\title{
Loop-Mediated Isothermal Amplification (LAMP) for the Detection of Listeria monocytogenes and Major Pathogenic Serotypes
}

\author{
Ana Paula Rocha da Costa, Mariana de Lira Nunes, Carina Lucena Mendes-Marques, \\ Alzira Maria Paiva de Almeida, Nilma Cintra Leal \\ Departamento de Microbiologia, Centro de Pesquisas Aggeu Magalhães, FIOCRUZ-PE, Recife, Brazil \\ Email: nilma@cpqam.fiocruz.br
}

Received 31 August 2014; revised 13 October 2014; accepted 31 October 2014

Copyright (C) 2014 by authors and Scientific Research Publishing Inc.

This work is licensed under the Creative Commons Attribution International License (CC BY). http://creativecommons.org/licenses/by/4.0/

(c) (i) Open Access

\begin{abstract}
Rapid identification and characterization of Listeria monocytogenes are required for the food industry, epidemiological studies, and disease prevention and control. However, typing procedures are labor-intensive and time-consuming, and they require technical expertise, a panel of sera and reference culture strains or sophisticated and expensive equipment. To improve upon traditional diagnostic methods for $L$. monocytogenes we developed and evaluated an efficient procedure for the specific identification of $L$. monocytogenes and the major pathogenic serotypes of the species based on loop-mediated isothermal amplification (LAMP). Four individual reactions were designed using primers targeting any L. monocytogenes serotypes (LAMP-AS) and the 1/2a (LAMP$1 / 2 a), 1 / 2 b$ (LAMP-1/2b), and $\underline{4 b}$ (LAMP-4b) serotypes. The procedure distinguished L. monocytogenes from closely genetically related species and the targeted serotypes. Cross-reactivity with a few rare serotypes isolated from food or clinical samples did not impair the usefulness of the procedure. Thus, our approach constitutes a fast, easy and low-cost alternative for $L$. monocytogenes diagnosis and serotyping and may be useful for surveillance and epidemiological investigation programs.
\end{abstract}

\section{Keywords}

Listeria monocytogenes, Diagnosis, Serotypes, Identification, LAMP 


\section{Introduction}

Listeria monocytogenes is a facultative intracellular food-borne pathogen that causes listeriosis, a disease of varying severity that poses a problem mainly for immune compromised individuals, pregnant women, newborns and the elderly [1].

The detection and identification of L. monocytogenes require culturing, morphological and biochemical tests, and serotyping by agglutination or immunoenzymatic assays (ELISA). Molecular studies have identified genetic markers specifically associated with phylogenetic groups, allowing for the development of several DNA-based diagnoses and typing procedures [2]-[4].

Based on $\mathrm{O}$ (somatic) and H (flagellar) antigens, 12 L. monocytogenes serotypes have been identified. These serotypes are grouped into three genetic lineages according to specific genetic sequences: lineage I includes the 4b, 1/2b, 3b, 4d, 4e and 7 serotypes; lineage II includes the 1/2a, 1/2c, 3a and 3c serotypes; and lineage III includes the 4a and 4c serotypes. Lineage I contains the major L. monocytogenes epidemic clones that are responsible for most cases of human listeriosis. Lineage II isolates come mainly from food and the environment, and lineage III isolates originate primarily from animals [5]-[7]. Therefore, at least 95\% of L. monocytogenes isolates from food and patient samples belong to three serotypes (1/2a, 1/2b and 4b), which are considered the most pathogenic [1].

The identification and characterization of $L$. monocytogenes are required for epidemiological studies to track and determine sources of infection, for food industry and safety, and for disease prevention and control. However, the routine methods used to detect L. monocytogenes are labor-intensive, time-consuming and expensive, and employ a panel of sera and reference culture strains usually available on reference laboratories only.

The loop-mediated isothermal amplification (LAMP) technology amplifies DNA with high efficiency using the Bst DNA polymerase under isothermal conditions. The technique employs a set of primers designed to recognize distinct regions of the same target gene, increasing the amplification specificity [8]. The procedure is quite simple and easy to perform. It only requires a water bath or laboratory heating block, and the amplifications can be visually determined by observing color changes with the naked eye or under ultraviolet (UV) [9].

To improve upon traditional diagnostic methods for $L$. monocytogenes we tested three major pathogenic serotypes of the species in LAMP amplification reactions with four different primer sets, named LAMP-AS for any L. monocytogenes serotypes, and LAMP-1/2a, LAMP-1/2b, and LAMP-4b for 1/2a, $\underline{1 / 2 b}$, and $\underline{4 b}$ L. monocytogenes serotypes respectively. Positive results were accordingly obtained in all cases. This LAMP-based approach has great potential for diagnosis and serotyping of L. monocytogenes and may contribute to the control of L. monocytogenes-associated disease.

\section{Experimental Procedures}

\subsection{Bacterial Strains}

The study involved 14 L. monocytogenes strains of different serotypes and the L. innoccua (CIP12612), L. ivanovii (ATCC19119), L. seeligeri (CIP8493), and L. welshimeri (CIP11633) species provided by the Laboratório de Zoonoses Bacterianas, Instituto Oswaldo Cruz (IOC/FIOCRUZ). The cultures used in the study are shown in Table 1. The assays used for standardization of the LAMP reactions employed the L. monocytogenes strains, CDCF4561 for serotype 1/2a, CDCF4976 for serotype 1/2b and ATCC19115 for serotype 4b, and the three strains in the reactions for any L. monocytogenes serotype. The culture conditions and DNA extraction were performed as previously described [10]. DNA concentration and purity were determined by spectrophotometry at 260/280 nm using a Nano Drop (Thermo Scientific).

\subsection{Design of the LAMP Primers}

The LAMP primers were designed according to previous studies [9]. Four sets of LAMP primers were designed for the specific identification of L. monocytogenes and the major pathogenic serotypes of the species, based on published primer sequences [2]-[4] targeting L. monocytogenes of any serotype (lmo2234) and targeting serotypes 1/2a (lmo0737), 1/2b (gltb) and 4b (ORF2110) (Table 2). Each primer set included two outer primers, a forward outer primer (F3) and a backward outer primer (B3), and two internal primers, a forward inner primer (FIP) and a backward inner primer (BIP).

LAMP primers were constructed using the Primer Explorer V4 software (http://primerexplorer.jp/) taking into consideration GC (guanine-cytosine) content, Tm (temperature dissociation), stability of the final sequence of 
Table 1. Strains used in this study.

\begin{tabular}{ccc}
\hline Strain & \multicolumn{1}{c}{ Species } & Serotype \\
\hline CDCF4561 & L. monocytogenes & $1 / 2 \mathrm{a}$ \\
CDCF4976 & L. monocytogenes & $1 / 2 \mathrm{~b}$ \\
ATCC19115 & L. monocytogenes & $4 \mathrm{~b}$ \\
CDCF6254 & L. monocytogenes & $1 / 2 \mathrm{c}$ \\
ATCC19112 & L. monocytogenes & $1 / 2 \mathrm{c}$ \\
CDCF45555 & L. monocytogenes & $4 \mathrm{~b}$ \\
ATCC19144 & L. monocytogenes & $4 \mathrm{a}$ \\
CDCF1067 & L. monocytogenes & $4 \mathrm{ab}$ \\
ATCC19116 & L. monocytogenes & $4 \mathrm{c}$ \\
ATCC19117 & L. monocytogenes & $4 \mathrm{~d}$ \\
ATCC19118 & L. monocytogenes & $4 \mathrm{e}$ \\
CDCF4540 & L. monocytogenes & $3 \mathrm{a}$ \\
CDCF4538 & L. monocytogenes & $3 \mathrm{~b}$ \\
CDCF6238 & L. monocytogenes & $3 \mathrm{c}$ \\
CIP12612 & L. innocua & Not applied \\
ATCC19119 & L. ivanovii & Not applied \\
CIP8493 & L. weeligeri & Not applied \\
CIP11633 & & Not applied
\end{tabular}

Table 2. LAMP primer sets.

\begin{tabular}{cccc}
\hline Primer set & Primer type & Sequence (5' to 3') & Target \\
\hline & F3 & GGCAATCTAAAAAAATGCCCCA & (isteria monocytogenes \\
(any serotype)
\end{tabular}

LAMP-AS: specific primers to any serotype L. monocytgenes; LAMP-1/2a: specific primers to L. monocytgenes serotype 1/2a; LAMP-1/2b: specific primers to L. monocytgenes serotype $1 / 2 \mathrm{~b}$; LAMP-4b: specific primers to L. monocytgenes serotype $4 \mathrm{~b}$; F3: forward outer primer; B3: backward outer primer; FIP: forward inner primer; BIP: backward inner primer.

the primers, secondary structure formation and distance between the primers [9]. Integrated DNA Technologies (IDT) synthesized desalinized primers. Primers were named LAMP-AS (lmo2234) for any L. monocytogenes serotypes, and LAMP-1/2a (lmo0737), LAMP-1/2b (gltb), and LAMP-4b (ORF2110) for $\underline{1 / 2 \mathrm{a}}, \underline{1 / 2 \mathrm{~b}}$, and $\underline{\mathrm{b}} \mathrm{L}$. monocytogenes serotypes respectively. 


\subsection{Standardization of the LAMP Reactions and Analysis of the Amplified Products}

Optimization of the reaction conditions for each set of primers and DNA template from the different targets was performed following a protocol developed by Tomita et al. [11]. In short, reactions were made in $25 \mu \mathrm{l}$ of reaction mixture composed of $50 \mathrm{pmol}$ of inner primer, $5 \mathrm{pmol}$ of outer primer, $20 \mathrm{mM}$ Tris- $\mathrm{HCl}, \mathrm{pH} 8.8,10 \mathrm{mM}$ $\left(\mathrm{NH}_{4}\right) \cdot 2 \mathrm{SO}_{4}, 8 \mathrm{mM} \mathrm{MgSO}$, $10 \mathrm{mM} \mathrm{KCl}, 1.4 \mathrm{mM}$ dNTPs, $0.8 \mathrm{M}$ betaine, $0.1 \%$ Tween 20, 8 units of Bst DNA polymerase (New Biolabs Engle) and 20 ng of DNA template. Reactions were performed individually for each set of primers described in Table 2. In each amplification run, a negative control containing all reaction components except template DNA was included. To determine the minimum incubation time for visualization of the amplified products, assays were performed at increasing incubation times (15, 30, 45, 60, 75 and 90 minutes) using a thermocycler (Biometra) and water bath in parallel. All reactions were performed at $63^{\circ} \mathrm{C}$, as satisfactory LAMP can be achieved at isothermal conditions between $60^{\circ} \mathrm{C}-65^{\circ} \mathrm{C}$ [12].

To evaluate the best mode of visualization, the products were stained with the fluorescent dyes, SYBR Safe ${ }^{\circledR}$ (Invitrogen ${ }^{\mathrm{TM}}$ ) for inspection by the naked eye and SYBR Green ${ }^{\circledR}$ (Promega) for inspection under UV. For purposes of comparison, the products were analyzed under UV after electrophoresis on 1\% agarose gels and staining with SYBR Green ${ }^{\circledR}$ (Promega).

\subsection{Determination of LAMP Sensitivity}

LAMP sensitivity was determined in PCR assays with each set of primers using serial dilutions (200 ng to 1 pg) of DNA from cultures of $L$. monocytogenes serotypes 1/2a (CDCF4561), 1/2b (CDCF4976) and 4b (ATCC19115). To determine the minimal detectable colony forming units (CFUs), cultures of each of the above strains were serially diluted $\left(10^{-1}\right.$ to $\left.10^{-6}\right)$, and aliquots of $100 \mu$ l of each dilution were plated in duplicate on brain heart infusion (BHI) agar. CFUs were counted after $24 \mathrm{~h}$ at $37^{\circ} \mathrm{C}$. PCR assays with each set of primers and the serial dilution $\left(10^{-1}\right.$ to $\left.10^{-6}\right)$ of each culture were then performed.

\subsection{Specificity of the LAMP Procedure}

To determine the specificity of the reactions, assays were performed in triplicate with each set of primers and template DNA from L. innoccua (CIP12612), L. ivanovii (ATCC19119), L. seeligeri (CIP8493), L. welshimeri (CIP11633) and 14 L. monocytogenes strains of several serotypes. Table 1 shows the strains used in this study.

\section{Results}

\subsection{Standardization of LAMP Reaction Conditions and Visualization of Amplified Products}

Three L. monocytogenes strains, serotypes 1/2a (CDCF4561), 1/2b (CDCF4976) and 4b (ATCC19115) were tested with four different primer sets (LAMP-AS for any L. monocytogenes serotypes, and LAMP-1/2a, LAMP$1 / 2$ and LAMP-4b for $\underline{1 / 2 \mathrm{a}}, \underline{1 / 2 \mathrm{~b}}$ and $\underline{4 \mathrm{~b}}$ L. monocytogenes serotypes respectively) in 12 reactions (Table 3 ).

Assays were performed using the four sets of primers (LAMP-AS, LAMP-1/2a, LAMP-1/2b, LAMP-4b) and using DNA from L. monocytogenes strains CDCF4561 (serotype 1/2a), CDCF4976 (serotype 1/2b) and ATCC19115 (serotype 4b). Clear and reproducible amplification products visualization was only achieved at 60 minutes or higher incubation time. Below this time amplification products visualization was unsatisfactory. Identical results were obtained in parallel assays using a thermo cycler and water bath. Therefore, the experiments proceeded using a water bath and 60 minutes incubation.

The established conditions allowed visualization of the amplified products under UV light upon the addition of SYBR Safe $^{\circledR}$ (Invitrogen ${ }^{\mathrm{TM}}$ ) (Figure 1(a)) or visualizing the products by the naked eye by observing a color change from orange to green upon the addition of SYBR Green ${ }^{\circledR}$ (Promega) (data not shown). The amplification was confirmed by visualizing the products on $1 \%$ agarose gels stained with SYBR Safe ${ }^{\circledR}$ (Invitrogen ${ }^{\mathrm{TM}}$ ) (Figure 1 (b)).

\subsection{Sensitivity of the LAMP Technique}

In assays with the four sets of primers and eight dilutions containing $200 \mathrm{ng}$ to $1 \mathrm{pg}$ of purified DNA from the $L$. monocytogenes strains, CDCF4561 (1/2a), CDCF4976 (1/2b) and ATCC19115 (4b), the LAMP detection limit was $100 \mathrm{pg}$ of purified DNA per reaction (data not shown). 
Table 3. Result of the LAMP reactions with the four LAMP primer sets (LAMP-1/2a, LAMP-1/2b, LAMP-4b, LAMP-AS) and the L. monocytogenes strains, serotypes 1/2a (CDCF4561), 1/2b (CDCF4976) and 4b (ATCC19115).

\begin{tabular}{|c|c|c|c|}
\hline LAMP primer & Gene target (serotype) & Strains (serotype) & LAMP \\
\hline \multirow{3}{*}{ LAMP-1/2a } & \multirow{3}{*}{$\operatorname{lmo} 0737(1 / 2 a)$} & CDCF4561 (1/2a) & + \\
\hline & & CDCF4976 (1/2b) & - \\
\hline & & ATCC19115 (4b) & - \\
\hline \multirow{3}{*}{ LAMP-1/2b } & \multirow{3}{*}{ Gltb (1/2b) } & CDCF4561 (1/2a) & - \\
\hline & & CDCF4976 (1/2b) & + \\
\hline & & ATCC19115 (4b) & - \\
\hline \multirow{3}{*}{ LAMP-4b } & \multirow{3}{*}{ ORF2110 (4b) } & CDCF4561 (1/2a) & - \\
\hline & & CDCF4976 (1/2b) & - \\
\hline & & ATCC19115 (4b) & + \\
\hline \multirow{3}{*}{ LAMP-AS } & \multirow{3}{*}{ lmo2234 (any serotype) } & CDCF4561 (1/2a) & + \\
\hline & & CDCF4976 (1/2b) & + \\
\hline & & ATCC19115 (4b) & + \\
\hline
\end{tabular}

LAMP-1/2a: specific primers to L. monocytgenes serotype $1 / 2 \mathrm{a} ;$ LAMP-1/2b: specific primers to L. monocytgenes serotype $1 / 2 \mathrm{~b}$; LAMP-4b: specific primers to L. monocytgenes serotype $\underline{4 b}$; LAMP-AS: specific primers to any serotype L. monocytgenes; + = positive (with amplification); - = negative (no amplification).

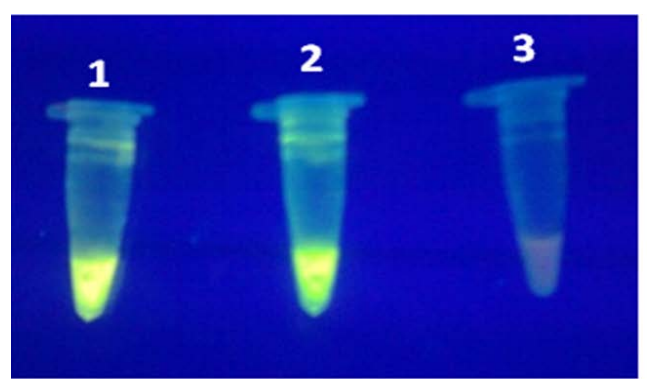

(a)

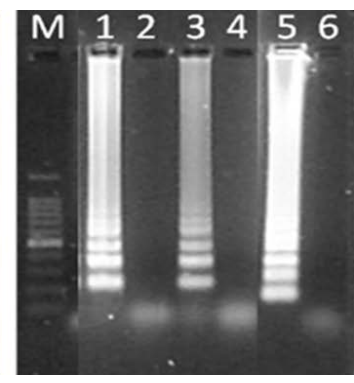

(b)

\begin{abstract}
Figure 1. (a) Visualization of LAMP products stained with SYBR ${ }^{\circledR}$ Green and inspected under UV. Tubes 1 and 2, positive reactions and tube 3 negative reaction; (b) $1 \%$ agarose gel electrophoretogram of LAMP products. Lines M: 100 bp molecular marker, 1: L. monocytogenes serotype 1/2a, 3: L. monocytogenes serotype 1/2b, 5: L. monocytogenes serotype $4 \mathrm{~b}$, lines 2, 4, 6 : negative controls.
\end{abstract}

In assays with the four sets of primers and six dilutions containing 10 to $10^{-5}$ CFUs from the CDCF4561 (1/2a), CDCF4976 (1/2b) and ATCC19115 (4b) strains, the LAMP detection limit was $10^{-4}$ CFUs per reaction with the three strains (data not shown).

\title{
3.3. Specificity of the LAMP
}

In assays with the LAMP-AS primer and DNA from the 14 L. monocytogenes strains of several serotypes (Table 1 ) or from the L. innoccua (CIP12612), L. ivanovii (ATCC19119), L. seeligeri (CIP8493), and L. welshimeri (CIP11633) species, amplification was detected only using DNA from L. monocytogenes. No amplification was observed with the other species of Listeria evaluated. Primer LAMP-1/2a, which is specific for serotype 1/2a, amplified the specific target as well as serotypes 1/2c, 3a and 3c; LAMP-1/2b, which is specific for serotype $1 / 2 b$, amplified the specific target as well as serotype 3b; and primer LAMP-4b, which is specific for serotype 4b, amplified the specific target as well as serotypes 4d and 4e. Other L. monocytogenes serotypes or Listeria species were not amplified.

\section{Discussion}

Although rare, listeriosis is a serious public health problem because it results in high mortality rates. Ingestion of 
contaminated food is considered the primary source of infection in both sporadic cases and inlisteriosis epidemics [1]. Infection with L. monocytogenes causes non-specific symptoms, such as meningitis and septicemia. Thus, the development of rapid diagnostic tests that are sensitive and specific for $L$. monocytogenes is important for selecting appropriate treatment regimens. Moreover, as L. monocytogenes consists of strains of varying pathogenicities, the ability to identify a virulent trains found in food versus pathogens involved in listeriosis outbreaks is crucial for the control and prevention of the disease [13].

Among the 12 serotypes of $L$. monocytogenes identified, only three are commonly isolated from clinical cases and foods: serotype $4 \mathrm{~b}$ is primarily responsible for clinical cases and outbreaks of listeriosis, and serotypes 1/2a and 1/2b are associated with sporadic infections and are the main serotypes found in food [7] [13] [14].

After identifying the causative organism in clinical or food samples, it is necessary to perform serological classification. However, serotyping is not possible in all clinical laboratories, and additionally, the reliability of the serotyping performed in many clinics is limited by the lack of standardized reagents and by the frequent lack of the necessary technical expertise among clinic staff. Kits for enzyme immunoassays and PCR-based protocols for the identification of pathogenic serotypes have overcome some of the standardization difficulties, but these kits are also expensive and require sophisticated equipment to perform the assays and read the assay results, hindering the adoption of these standardized kits and protocols in many field laboratories [2]-[4] [15].

The LAMP technique is easier to perform than some of the standardized assays because it requires only a water bath and the reaction components. LAMP also has the advantage of reduced reaction times. The preparation of a LAMP or PCR mix is simpler than performing a serological test, as once the kit components are combined, the operator only needs to add the DNA or culture sample.

The LAMP procedure generally employs a set of four to six primers comprising two outer, two inner and two loop primers that recognize six to eight distinct regions of the target sequence. The two outer primers, referred to as the forward (F3) and backward (B3) outer primers in the study, play a role in strand displacement during the non-cyclic step. The internal primers, the forward (FIP) and backward (BIP) internal primers in the study, have both sense and antisense sequences and participate in the formation of a loop. Finally, two loop primers, referred to as the forward (FLP) and backward (BLP) loop primers in the study, accelerate the amplification reaction by binding to additional sites that are not accessed by the internal primers [9].

We did not include the optional loop primers in the study, as we wanted to further simplify and cut the costs of the procedure. Even without the optional primers, satisfactory amplification was achieved in as fast as 60 minutes. All reactions were performed at $63^{\circ} \mathrm{C}$, as previous reports have indicated that isothermal conditions between $60^{\circ} \mathrm{C}-65^{\circ} \mathrm{C}$ give similar results in LAMP assays [12].

LAMP assays for diagnosing L. monocytogenes infection have previously been reported [12] [16] [17]. Although these techniques are more sensitive than the LAMP we present here, none of them intended species detection with serotypes distinction. A novel aspect of this paper is that our approach aimed to specifically identify $L$. monocytogenes as well as the major pathogenic serotypes of the species using the following specific primers: LAMP-AS for L. monocytogenes regardless of serotype, LAMP-1/2a for serotype 1/2a, LAMP-1/2b for serotype $1 / 2 \mathrm{~b}$ and LAMP-4b for serotype $4 \mathrm{~b}$.

The specificity of the LAMP is directly attributed to the set of primers spanning several sequences of the gene targeted for amplification. Hence, unless the target is available, amplification will not proceed [9].

The LAMP procedure described herein permitted the differentiation of L. monocytogenes from closely genetically related species. As limitation, cross-reactivity occurred between the LAMP-1/2a primer for serotype 1/2a and the 1/2c, 3a, and 3c serotypes; between the LAMP-1/2b primer for serotype 1/2b and the 3b serotype; and between the LAMP- $4 \mathrm{~b}$ primer for serotype $4 \mathrm{~b}$ and the $4 \mathrm{~b}$ and $4 \mathrm{e}$ serotypes. These results are due to the presence of $\mathrm{H}$ and $\mathrm{O}$ antigens and gene sequences shared by the serotypes [13] [14]. Thus, the genetic sequences employed in the current study are shared between closely related serotypes: lmo0737 (LAMP-1/2a) is shared among serotypes $1 / 2 \mathrm{a}, 1 / 2 \mathrm{c}$, 3a and 3c [3], gltb (LAMP-1/2b) is shared between serotypes $1 / 2 \mathrm{~b}$ and 3b [2] and ORF2110 (LAMP-4b) is shared among serotypes 4b, 4d and 4e [4].

However, this cross-reactivity among the above serotypes did not impair the LAMP technique, as serotypes 3a, 3b, 4d and 4e are rarely isolated from food or patients. According to data collected by the National Reference Center in France, more than $98 \%$ of the 5000 L. monocytogenes isolates obtained from food and patient samples between 2001 and 2004 were serotypes 1/2a, 1/2b, 1/2c, and 4b. These serotypes were able to be categorized into three groups by the present LAMP standardized technique.

Other authors reported that LAMP is more sensitive and less influenced by components of biological samples 
than conventional PCR and real time PCR [12] [16] [17]. Although we did not compare herein the LAMP with other technologies, one can expect that our protocol would be successfully applied for biological samples.

\section{Conclusion}

We conclude that the LAMP technique standardized in this study represents a quick and easy alternative to serological typing and may be used for the diagnosis of L. monocytogenes, especially for surveillance and epidemiological research.

\section{Acknowledgements}

We acknowledge the Laboratório de Zoonoses Bacterianas, Instituto Oswaldo Cruz (IOC/FIOCRUZ), RJ, Brazil for providing the strains used in this study.

\section{References}

[1] Swaminathan, B. and Gerner-Smidt, P. (2007) The Epidemiology of Human Listeriosis. Microbes and Infection, 9, 1236-1243. http://dx.doi.org/10.1016/j.micinf.2007.05.011

[2] Borucki, M.K. and Call, D.R. (2003) Listeria monocytogenes Serotype Identification by PCR. Journal of Clinical Microbiology, 41, 5537-5540. http://dx.doi.org/10.1128/JCM.41.12.5537-5540.2003

[3] Chen, Y. and Knabel, S.J. (2007) Multiplex PCR for Simultaneous Detection of Bacteria of the Genus Listeria, Listeria monocytogenes, and Major Serotypes and Epidemic Clones of L. monocytogenes. Applied and Environmental Microbiology, 73, 6299-6304. http://dx.doi.org/10.1128/AEM.00961-07

[4] Doumith, M., Buchrieser, C., Glaser, P., Jacquet, C. and Martin, P. (2004) Differentiation of the Major Listeria monocytogenes Serovars by Multiplex PCR. Journal of Clinical Microbiology, 42, 3819-3822. http://dx.doi.org/10.1128/JCM.42.8.3819-3822.2004

[5] Brosch, R., Chen, J. and Luchansky, J.B. (1994) Pulsed-Field Fingerprinting of Listeriae: Identification of Genomic Divisions for Listeria monocytogenes and Their Correlation with Serovar. Applied and Environmental Microbiology, 60, 2584-2592.

[6] Cossart, P. (2011) Illuminating the Landscape of Host-Pathogen Interactions with the Bacterium Listeria monocytogenes. Proceedings of the National Academy of Sciences of the United States of America, 108, 19484-19491. http://dx.doi.org/10.1073/pnas.1112371108

[7] Wiedmann, M., Bruce, J.L., Keating, C., Johnson, A.E., McDonough, P.L. and Batt, C.A. (1997) Ribotypes and Virulence Gene Polymorphisms Suggest Three Distinct Listeria monocytogenes Lineages with Differences in Pathogenic Potential. Infection and Immunity, 65, 2707-2716.

[8] Notomi, T., Okayama, H., Masubuchi, H., Yonekawa, T., Watanabe, K., Amino, N. and Hase, T. (2000) LoopMediated Isothermal Amplification of DNA. Nucleic Acids Research, 28, E63. http://dx.doi.org/10.1093/nar/28.12.e63

[9] Parida, M., Sannarangaiah, S., Dash, P.K., Rao, P.V.L. and Morita, K. (2008) Loop Mediated Isothermal Amplification (LAMP): A New Generation of Innovative Gene Amplification Technique; Perspectives in Clinical Diagnosis of Infectious Diseases. Reviews in Medical Virology, 18, 407-421. http://dx.doi.org/10.1002/rmv.593

[10] Costa, A.P.R., Vilela, M.A., Mendes-Marques, C.L., Almeida, A.M.P. and Leal, N.C. (2013) Biochemical and Molecular Characteristics of Listeria monocytogenes Isolates from a Prosthetic Mitral Heart Valve-Bearing Patient's Blood Cultures. International Journal of Biological Sciences, 1, 116-121.

[11] Tomita, N., Mori, Y., Kea, H. and Notomi, T. (2008) Loop-Mediated Isothermal Amplification (LAMP) of Gene Sequences and Simple Visual Detection of Products. Nature Protocols, 3, 877-882. http://dx.doi.org/10.1038/nprot.2008.57

[12] Wang, L., Li, Y., Chu, J., Xu, Z. and Zhong, Q. (2012) Development and Application of a Simple Loop-Mediated Isothermal Amplification Method on Rapid Detection of Listeria monocytogenes Strains. Molecular Biology Reports, 39, 445-449. http://dx.doi.org/10.1007/s11033-011-0757-7

[13] Liu, D. (2006) Identification, Subtyping and Virulence Determination of Listeria monocytogenes, an Important Foodborne Pathogen. Journal of Medical Microbiology, 55, 645-659. http://dx.doi.org/10.1099/jmm.0.46495-0

[14] Schönberg, A., Bannermanb, E., Courtieuc, A.L., Kissd, R., McLauchlin, J., Shah, S. and Wilhelms, D. (1996) Serotyping of 80 Strains from the WHO Multicentre International Typing Study of Listeria monocytogenes. International Journal of Food Microbiology, 32, 279-287. http://dx.doi.org/10.1016/S0168-1605(96)01142-7

[15] Palumbo, J.D., Borucki, M.K., Merell, R.E. and Gorski, L. (2003) Serotyping of Listeria monocytogenes by EnzymeLinked Immunosorbent Assay and Identification of Mixed-Serotype Cultures by Colony Immunoblotting. Journal of 
Clinical Microbiology, 41, 564-571. http://dx.doi.org/10.1128/JCM.41.2.564-571.2003

[16] Tang, M.J., Zhou, S., Zhang, X.Y., Pu, J.H., Ge, Q.L., Tang, X.J. and Gao, Y.S. (2011) Rapid and Sensitive Detection of Listeria monocytogenes by Loop-Mediated Isothermal Amplification. Current Microbiology, 63, 511-516. http://dx.doi.org/10.1007/s00284-011-0013-3

[17] Cho, A.R., Dong, H.J., Seo, K.H. and Cho, S. (2014) Development of a Loop-Mediated Isothermal Amplification Assay for Detecting Listeria monocytogenes prfA in Milk. Food Science and Biotechnology, 23, 467-474. http://dx.doi.org/10.1007/s10068-014-0064-x

\section{List of Abbreviations}

LAMP: loop-mediated isothermal amplification;

LAMP-AS: specific primers to any serotype L. monocytgenes;

LAMP-1/2a: specific primers to $L$. monocytgenes serotype $\underline{1 / 2 \mathrm{a}}$;

LAMP-1/2b: specific primers to L. monocytgenes serotype $\underline{1 / 2 b}$;

LAMP-4b: specific primers to L. monocytgenes serotype $\underline{4 \mathrm{~b}}$;

F3: forward outer primer;

B3: backward outer primer;

FIP: forward inner primer;

BIP: backward inner primer;

UV: ultraviolet;

CFU: colony forming units;

BHI: brain heart infusion agar. 
Scientific Research Publishing (SCIRP) is one of the largest Open Access journal publishers. It is currently publishing more than 200 open access, online, peer-reviewed journals covering a wide range of academic disciplines. SCIRP serves the worldwide academic communities and contributes to the progress and application of science with its publication.

Other selected journals from SCIRP are listed as below. Submit your manuscript to us via either submit@scirp.org or Online Submission Portal.
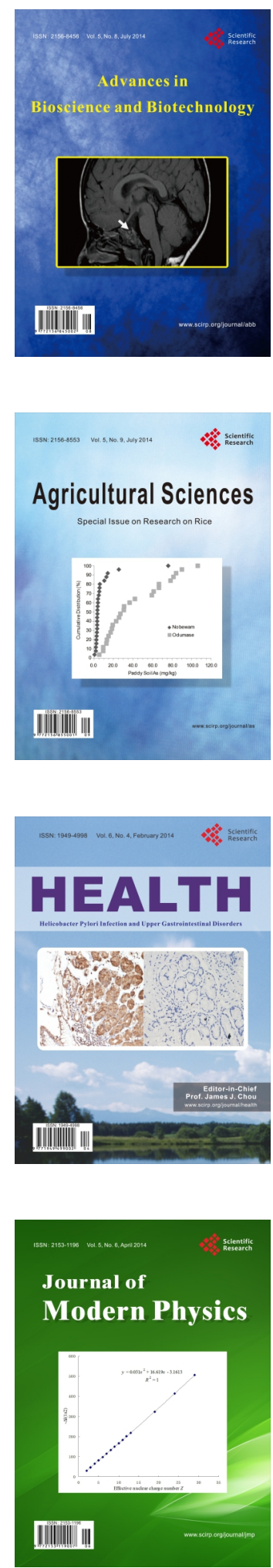
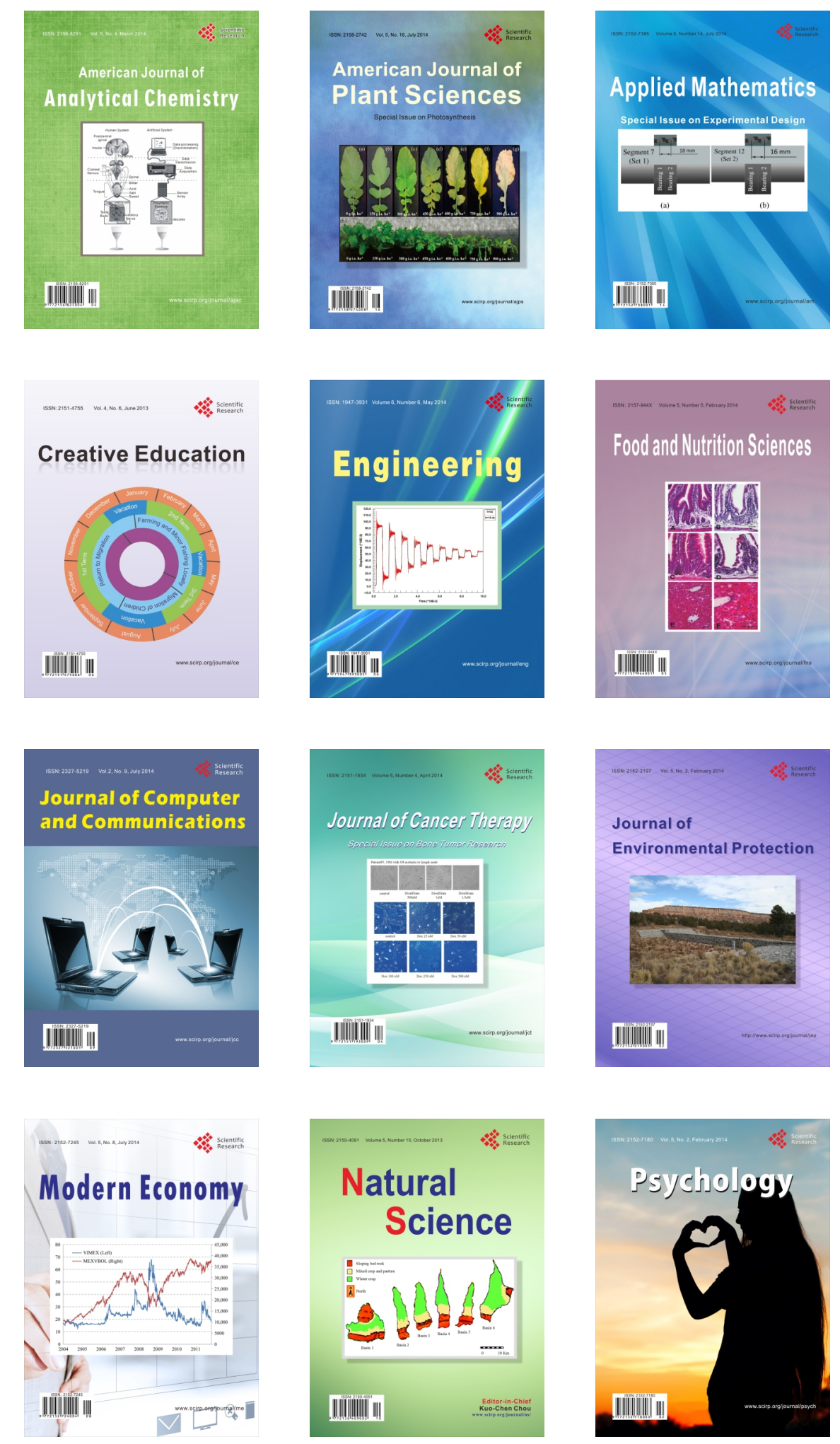